\title{
Periodic Solutions Generated by Impulses for State-Dependent Impulsive Differential Equation
}

\author{
Qizhen Xiao and Binxiang Dai \\ School of Mathematics and Statistics, Central South University, Changsha, Hunan 410083, China \\ Correspondence should be addressed to Binxiang Dai; bxdai@csu.edu.cn
}

Received 6 August 2014; Accepted 26 September 2014

Academic Editor: Zhengrong Xiang

Copyright (C) 2015 Q. Xiao and B. Dai. This is an open access article distributed under the Creative Commons Attribution License, which permits unrestricted use, distribution, and reproduction in any medium, provided the original work is properly cited.

We study the existence of periodic solutions for a class of state-dependent impulsive differential systems via geometrical analysis methods. Our results show that these periodic solutions are generated by impulses. Moreover, numerical simulations are used to examine the existence of the periodic solutions.

\section{Introduction}

It is known that many evolutionary processes are characterized by the fact that at certain moments of time the states change abruptly. Such processes often occur in biology, control theory, optimization theory, physics, and mechanics problems (e.g., [1-6]). It is natural to assume that these perturbations act instantaneously, that is, in the form of impulses.

The theory of impulsive differential equations (IDEs) is rather rich, especially for impulse at fixed time. There are many classical methods to study impulsive differential equations. For example, Chen et al. [7] obtained some new results concerning the existence of solutions to an impulsive firstorder, nonlinear ordinary differential equation with periodic boundary conditions via differential inequalities and Schaefer's fixed-point theorem. Wang et al. [8] got the existence of extreme solutions of a periodic boundary value problem for a second-order functional differential equation by using upper and lower solutions. Based on a nonlinear alternative principle of Leray-Schauder, together with a truncation technique, Chu and Nieto [9] studied the impulsive periodic solutions of first-order singular ordinary differential equations. By using a variational method and a variant fountain theorem, Dai and Zhang [10] considered the existence and multiplicity of solutions for a class of nonlinear impulsive problem on the half-line. For more related work, the reader is referred to [11-13] and the references therein. As we know, state-dependent IDEs have become a hot topic in recent years due to their extensive application space, but it is also a difficult research field because of their essential properties: uncertainties for impulsive time and collision times. Very recently, many papers have been devoted to the analysis of IDEs with statedependent impulsive effect. By using differential equation geometry theory and the method of successor functions, the existence and stability of periodic solution for pest management model with state feedback control strategy were discussed in $[14,15]$ and the homoclinic cycle and homoclinic bifurcation were analyzed for predator-prey model with statedependent impulsive harvesting in $[16,17]$. On the basis of rotated vector fields theory, Dai et al. [18] discussed the order1 positive periodic solution and homoclinic cycles and homoclinic bifurcations for a general semicontinuous dynamic system. Considering the influence of Allee effect on prey species, the authors in $[19,20]$ investigated a prey-predator model with Allee effect and state-dependent impulsive harvesting and got the sufficient conditions for the existence of order-1 periodic solution and heteroclinic bifurcation via the geometry theory of semicontinuous dynamic systems. Some other related studies can be seen in [21-23] and the references therein.

The aforementioned papers all assumed that the predator just lived on the prey. However, in practice, it is very likely that many enemies have some other food sources. Motivated by this, in this paper, we consider the following state-dependent predator-prey model in which the predator species display the logistic growth in the absence of prey species:

$$
\begin{aligned}
& x^{\prime}(t)=x\left(r_{1}-a_{11} x-a_{12} y\right) \\
& y^{\prime}(t)=y\left(r_{2}+a_{21} x-a_{22} y\right) \\
& x \neq h_{1}, h_{2} \quad \text { or } \quad x=h_{1}, \quad y>\tilde{y},
\end{aligned}
$$




$$
\begin{array}{r}
\Delta x(t)=0, \quad \Delta y(t)=\tau_{1}, \quad x=h_{1}, \quad y \leq \tilde{y}, \\
\Delta x(t)=-\alpha x, \quad \Delta y(t)=-\beta y+\tau_{2}, \\
x=h_{2},
\end{array}
$$

where $x(t)$ and $y(t)$ denote population densities of prey and predator at time $t$, respectively. All the parameters are positive constants, in addition, $\alpha, \beta \in(0,1), h_{1}<h_{2}$, and $\left(h_{1}, \widetilde{y}\right)$ is the point of intersection of $x^{\prime}=0$ and $x=h_{1}$.

This paper is organized as follows. In Section 2, we present some preliminaries. Then in Section 3, we discuss the existence of positive periodic solution of system (1) for different cases. At last, in Section 4, some numerical simulations and conclusions are presented.

\section{Preliminaries}

For Model (1), if there is no impulsive effect, we have the following subsystem:

$$
\begin{aligned}
& x^{\prime}(t)=x\left(r_{1}-a_{11} x-a_{12} y\right), \\
& y^{\prime}(t)=y\left(r_{2}+a_{21} x-a_{22} y\right) .
\end{aligned}
$$

Followed by [24], the following results can be concluded.

Lemma 1. Consider Model (2), there are one trivial equilibrium $S_{0}=(0,0)$ and two boundary equilibria $S_{1}=\left(r_{1} / a_{11}, 0\right)$ and $S_{2}=\left(0, r_{2} / a_{22}\right) . S_{0}$ is always unstable; $S_{1}$ is a saddle. Moreover, if $r_{1} a_{22}-r_{2} a_{12}>0$, then $S_{2}$ is a saddle and there exists $a$ unique positive equilibrium

$$
S_{+}=\left(x^{*}, y^{*}\right)=\left(\frac{r_{1} a_{22}-r_{2} a_{12}}{a_{11} a_{22}+a_{12} a_{21}}, \frac{r_{2} a_{11}+r_{1} a_{21}}{a_{11} a_{22}+a_{12} a_{21}}\right) \text {, }
$$

which is globally asymptotically stable.

Throughout this paper, we always assume that the condition $r_{1} a_{22}-r_{2} a_{12}>0$ holds true. Considering the biological background, we only discuss Model (1) in the region $\{(x, y)$ : $x \geq 0, y \geq 0\}$. Obviously, due to Lakshmikantham et al. [25] and Bainov and Simeonov [26], the global existence and uniqueness of solution for Model (1) are guaranteed by the smoothness properties of right-side functions.

To discuss the dynamics of Model (1), we define three cross sections and two regions:

$$
\begin{gathered}
\Sigma_{0}=\left\{(x, y): x=(1-\alpha) h_{2}, y>0\right\} ; \\
\Sigma_{1}=\left\{(x, y): x=h_{1}, y>0\right\} ; \\
\Sigma_{2}=\left\{(x, y): x=h_{2}, y>0\right\} ; \\
\Omega_{1}=\left\{(x, y): x \leq h_{1}, y>0\right\} ; \\
\Omega_{2}=\left\{(x, y): x \leq h_{2}, y>0\right\} .
\end{gathered}
$$

Definition 2 (see [27]). Suppose that the impulse set $M$ and its phase set $N$ are both lines, as shown in Figure 1. Assume

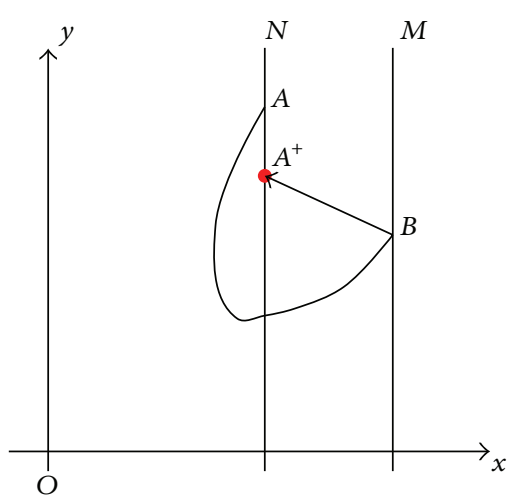

FIGURE 1: Illustration of the successor function.

that the trajectory starting from $A$ in $N$ firstly intersects $M$ at point $B$ and then jumps to $A^{+}$in $N$ due to the impulsive effect. Then, one defines $A^{+}$as the successor point of $A$, and the corresponding successor function of point $A$ is that $f(A)=$ $y_{A^{+}}-y_{A}$; here $y_{A}$ and $y_{A^{+}}$are the ordinates of $A$ and $A^{+}$.

Lemma 3 (see [27]). Successor function $f(A)$ is continuous.

Lemma 4 (see [27]). For Model (1), if there exist $\widetilde{A} \in N, \widehat{A} \in$ $N$ satisfying successor function $f(\widetilde{A}) f(\widehat{A})<0$, then there must exist a positive periodic solution.

\section{Existence of Positive Periodic Solution for System (1)}

Considering the biological meaning, here we always assume that $h_{1}<x^{*}$. Therefore, we have four cases to discuss: (1$\alpha) h_{2}<h_{1}<h_{2}<x^{*}, h_{1}<(1-\alpha) h_{2}<h_{2}<x^{*}, h_{1}<$ $(1-\alpha) h_{2}<x^{*}<h_{2}$, and $(1-\alpha) h_{2}<h_{1}<x^{*}<h_{2}$.

3.1. The Case of $(1-\alpha) h_{2}<h_{1}<h_{2}<x^{*}$. About the existence of positive periodic solution, we have the following graph illustrations.

Take a point $P\left(h_{1}, \tilde{y}+\varepsilon\right)$ on $\sum_{1}$, where $\varepsilon$ is small sufficiently. Assuming that the trajectory of Model (1) starting from $P$ firstly intersects $\sum_{1}$ at point $P_{1}$ and then jumps to $P_{1}^{+}$, obviously, $P_{1}^{+}$is above $P$; that is to say,

$$
f(P)=y_{P_{1}^{+}}-y_{P}>0
$$

On the other hand, assume that the trajectory starting from $P_{1}^{+}$intersects $\sum_{1}$ at $P_{2}$ and then jumps to $P_{2}^{+}$. For $P_{2}^{+}$, if $\tilde{y}<y_{P_{2}^{+}} \leq y_{P_{1}^{+}}$, then

$$
f\left(P_{1}^{+}\right)=y_{P_{2}^{+}}-y_{P_{1}^{+}} \leq 0
$$

thus, Model (1) exists as a positive periodic solution whose initial point is between $P$ and $P_{1}^{+}$; see Figure 2(a). If $y_{P_{2}^{+}} \leq \tilde{y}$, then Model (1) will keep on impulse until $y_{P_{2}^{k+}}>\tilde{y}\left(y_{P_{2}^{k+}}=\right.$ $\left.y_{P_{2}}+k \tau_{2}\right)$; this returns to the situation of $\tilde{y}<y_{P_{2}^{+}} \leq y_{P_{1}^{+}}$and the positive periodic solution can be seen in Figure 2(b). 


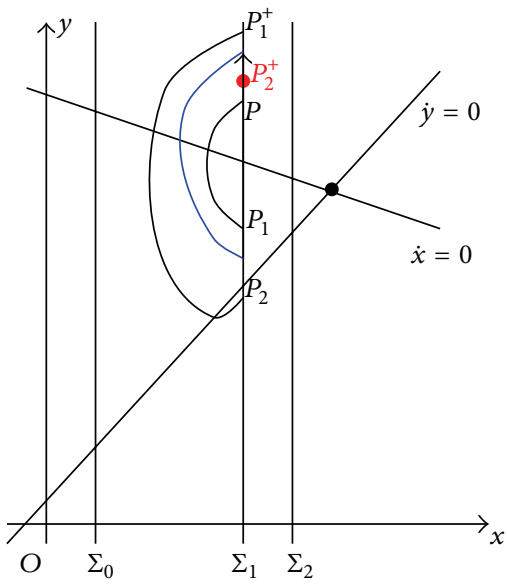

(a)

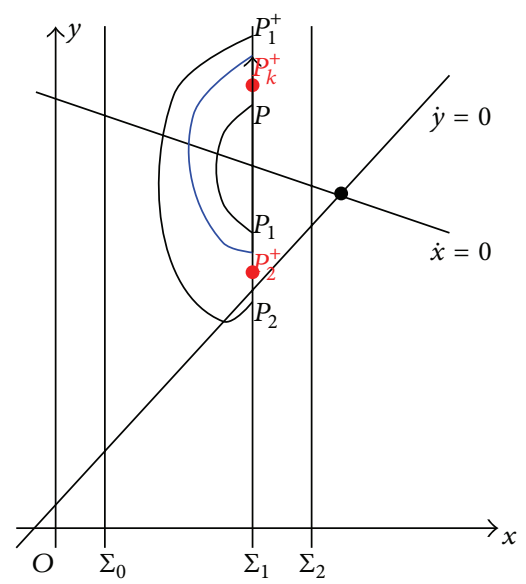

(b)

FIgURE 2: The possible trajectories in the case of $(1-\alpha) h_{2}<h_{1}<h_{2}<x^{*}$.

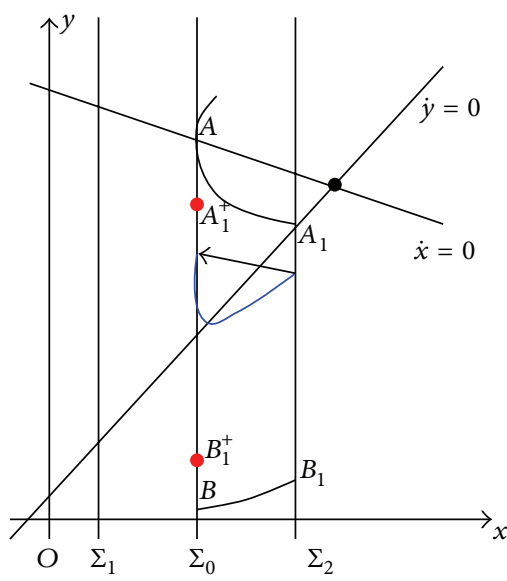

(a)

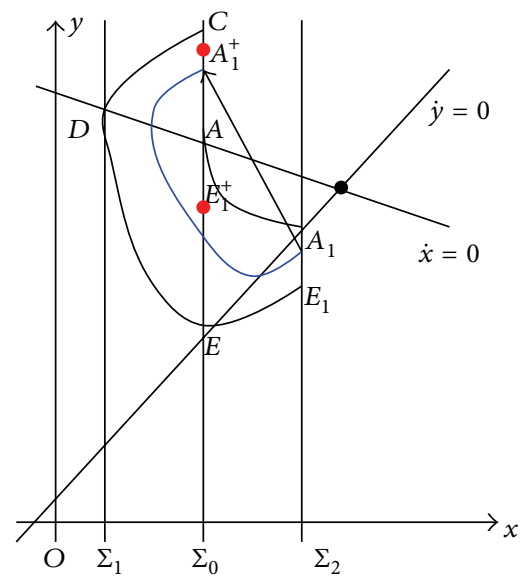

(b)

FIgURE 3: The possible trajectories in the case of $h_{1}<(1-\alpha) h_{2}<h_{2}<x^{*}$.

Theorem 5. Assume that $(1-\alpha) h_{2}<h_{1}<h_{2}<x^{*}$. Then Model (1) exists as a positive periodic solution whose initial point is located between $P$ and $P_{1}^{+}$.

3.2. The Case of $h_{1}<(1-\alpha) h_{2}<h_{2}<x^{*}$. For this case, we have the following graph illustrations.

Assuming that the trajectory starting from $A\left((1-\alpha) h_{2}, \hat{y}\right)$ firstly intersects $\sum_{2}$ at $A_{1}$ and then jumps to $A_{1}^{+}$, here $A$ is the intersection point of $x^{\prime}=0$ and $\Sigma_{0}$. For $A_{1}^{+}$, we have the following two situations.

(i) If $y_{A_{1}^{+}} \leq y_{A}$, then

$$
f(A)=y_{A_{1}^{+}}-y_{A} \leq 0 .
$$

On the other hand, choosing a point $B$ next to $x$-axis on $\sum_{0}$, the trajectory starting from $B$ firstly intersects $\sum_{2}$ at point $B_{1}$ and then jumps to $B_{1}^{+}$on $\sum_{0}$; obviously, $B_{1}^{+}$is above $B$; thus,

$$
f(B)=y_{B_{1}^{+}}-y_{B}>0 .
$$

Therefore, Model (1) exists as a positive periodic solution whose initial point is between $A$ and $B$; this is shown in Figure 3(a).

(ii) If $y_{A_{1}^{+}}>y_{A}$, then

$$
f(A)=y_{A_{1}^{+}}-y_{A}>0 .
$$

On the other hand, there must exist a trajectory starting from $C$ on $\sum_{0}$ that tangents $\sum_{1}$ at point $D\left(h_{1}, \widetilde{y}\right)$ and then intersects $\sum_{0}, \sum_{2}$ at points $E, E_{1}$, respectively; due to impulsive effect, $E_{1}$ jumps to $E_{1}^{+}$. For $E_{1}^{+}$, if $y_{E_{1}^{+}} \leq y_{C}$, then

$$
f(C)=y_{E_{1}^{+}}-y_{C} \leq 0 .
$$

Therefore, Model (1) exists as a positive periodic solution whose initial point is between $A$ and $C$; this can be seen in Figure 3(b). If $y_{E_{1}^{+}}>y_{C}$, then the trajectory starting from $\Omega_{2}$ will ultimately stay in $\Omega_{1}$. (Here we always assume the impulsive phase set with initial point on $\sum_{0}$ will ultimately 
exceed point $E$ after one or finite times impulses. In fact, the assumption is reasonable as $\beta$ should be very small in practical problem.)

Theorem 6. Assume that $h_{1}<(1-\alpha) h_{2}<h_{2}<x^{*}$. If $y_{A_{1}^{+}} \leq$ $y_{A}$ or $y_{A_{1}^{+}}>y_{A}, y_{E_{1}^{+}} \leq y_{C}$, then Model (1) exists as a positive periodic solution.

3.3. The Case of $h_{1}<(1-\alpha) h_{2}<x^{*}<h_{2}$. For this case, there must exist a trajectory $\Gamma$ starting from $A$ at $\Sigma_{2}$ that firstly intersects $\dot{x}=0, \dot{y}=0$ at $B, C$, respectively, and then tangents $\Sigma_{2}$ at point $D$. For $x_{B}$, there are three possible situations.

3.3.1. $(1-\alpha) h_{2}<x_{B}<h_{2}$. Obviously, there must exist a trajectory $\Gamma_{1}$ starting from $A_{1}$ at $\Sigma_{2}$ that tangents $\Sigma_{0}$ at point $B_{1}$ and then intersects $y^{\prime}=0, \Sigma_{2}$ at $C_{1}, D_{1}$, respectively. Due to impulsive effect, $D_{1}$ jumps to $D_{1}^{+}$. For $D_{1}^{+}$, there are two cases.

(i) If $y_{D_{1}^{+}} \leq y_{B_{1}}$, obviously, this situation returns to (i) of case of Section 3.2; here we omit it.

(ii) If $y_{D_{1}^{+}}>y_{B_{1}}$, assume that the trajectory starting from $D_{1}^{+}$intersects $x^{\prime}=0, y^{\prime}=0$, and $\Sigma_{2}$ at $B_{2}, C_{2}$, and $D_{2}$, respectively. Obviously, for the orbit $\widehat{D_{1}^{+} \widehat{B_{2} C_{2}} D_{2}}, x_{B_{2}}$ is the smallest abscissa. For $x_{B_{2}}$, there are two possible positives.

(a) If $x_{B_{2}} \geq h_{1}$, then Model (1) exists as a positive periodic solution whose initial point is between $B_{1}$ and $D_{1}^{+}$; this is shown in Figure 4(a).

(b) If $x_{B_{2}}<h_{1}$, then there must exist a trajectory $\Gamma^{\prime}$ starting from $P$ at $\Sigma_{0}$ that tangents $\Sigma_{1}$ at point $P_{1}$ and then intersects $\Sigma_{0}$ and $\Sigma_{2}$ at points $P_{2}$ and $P_{3}$. For $P_{3}$, it also has two possibilities:

$(\text { a) })^{\prime}$ if $y_{P_{3}^{+}} \leq y_{P}$, then Model (1) exists as a positive periodic solution whose initial point is between $B_{1}$ and $P$; this can be seen in Figure 4(b);

(b) ${ }^{\prime}$ if $y_{P_{3}^{+}}>y_{P}$, then the trajectory starting from $\Omega_{0}=\{(x, y):(x, y) \in \widehat{A B C D} A\}$ will tend to equilibrium and the trajectory starting from $\Omega_{2} \backslash \Omega_{0}$ will ultimately stay in $\Omega_{1}$. (In this case, we still assume the impulsive phase set with initial point on $\sum_{0}$ will ultimately exceed point $P_{2}$ after one or finite impulses.)

Theorem 7. Assume that $h_{1}<(1-\alpha) h_{2}<x_{B}<x^{*}<h_{2}$. If $y_{D_{1}^{+}} \leq y_{B_{1}}$ or $y_{D_{1}^{+}}>y_{B_{1}}, x_{B_{2}} \geq h_{1}$ or $y_{D_{1}^{+}}>y_{B_{1}}, x_{B_{2}}<h_{1}$, $y_{P_{3}^{+}} \leq y_{P}$, then Model (1) exists as a positive periodic solution.

3.3.2. $h_{1}<x_{B}<(1-\alpha) h_{2}$. Assuming that the trajectory $\Gamma$ intersects $\Sigma_{0}$ at points $P_{1}, P_{2}$ with $y_{P_{1}}>y_{P_{2}}$, due to impulsive effect, $D$ jumps to $D^{+}$. For $D^{+}$, one has the following four situations to discuss.

(i) If $y_{D^{+}}=y_{P_{1}}$ or $y_{D^{+}}=y_{P_{2}}$, then $P_{1} \widehat{B C D P_{1}}$ or $\widehat{P_{2} D P_{2}}$ is an order-1 periodic solution. (ii) If $y_{D^{+}}<y_{P_{2}}$, then it is easy to get that there exists an order-1 periodic solution; here we omit the details.

(iii) If $y_{D^{+}}>y_{P_{1}}$, assume that the trajectory starting from $D^{+}$intersects $\dot{x}=0$ at point $E$. For $x_{E}$, there exist two cases.

(a) If $x_{E} \geq h_{1}$, then Model (1) exists as a positive periodic solution whose initial point is between $P_{1}$ and $D^{+}$(see Figure 5(a)).

(b) If $x_{E}<h_{1}$, then there must exist a trajectory $\Gamma_{1}$ starting from $P$ at $\Sigma_{2}$ that firstly intersects $\Sigma_{0}$ at point $A_{1}$ and then tangents to $\Sigma_{1}$ at point $B_{1}$, and intersects $y^{\prime}=0, \Sigma_{2}$ at points $C_{1}, D_{1}$, respectively. Due to impulsive effect, $D_{1}$ jumps to $D_{1}^{+}$. For $D_{1}^{+}$, we have the following two cases to discuss:

$\left(\right.$ a) ${ }^{\prime}$ if $y_{D_{1}^{+}} \leq y_{A_{1}}$, then Model (1) exists as a positive periodic solution whose initial point is between $P_{1}$ and $A_{1}$ (see Figure 5(b));

(b) ${ }^{\prime}$ if $y_{D_{1}^{+}}>y_{A_{1}}$, then the trajectory starting from $\Omega_{0}=\left\{(x, y):(x, y) \in A \widehat{A P_{1} B C D} A\right\}$ will tend to equilibrium and the trajectory starting from $\Omega_{2} \backslash \Omega_{0}$ will ultimately stay in $\Omega_{1}$.

(iv) If $y_{P_{2}}<y_{D^{+}}<y_{P_{1}}$, then the trajectory starting from $\Omega_{0}=\left\{(x, y):(x, y) \in P A_{1} \widehat{B_{1} O_{1}} O_{2} P\right\}\left(O_{i}\left(h_{i}, 0\right)(i=\right.$ $1,2)$ ) will tend to equilibrium and the trajectory starting from $\Omega_{2} \backslash \Omega_{0}$ will ultimately stay in $\Omega_{1}$.

Theorem 8. Assume that $h_{1}<x_{B}<(1-\alpha) h_{2}<x^{*}<h_{2}$. If $y_{D^{+}} \leq y_{P_{2}}$ or $y_{D^{+}} \geq y_{P_{1}}, x_{E} \geq h_{1}$ or $y_{D^{+}}>y_{P_{1}}, x_{E}<h_{1}$, $y_{D_{1}^{+}} \leq y_{A_{1}}$, then Model (1) exists as a positive periodic solution.

Similarly, for the case $h_{1}<(1-\alpha) h_{2}<x^{*}<h_{2}$ with $x_{B}<h_{1}$ or $(1-\alpha) h_{2}<h_{1}<x^{*}<h_{2}$, one can prove there is no positive periodic solution; here we omit it.

Remark 9. The positive periodic solutions for Model (1) obtained in Theorem 5 Theorem 8 are generated by impulses. Here, we say that a solution is generated by impulses if this solution is nontrivial when impulsive effect exists, but it is trivial when there does not exist impulsive effect. For example, when $r_{1} a_{22}-r_{2} a_{12}>0$, by Lemma 1 we know that Model (2) does not possess any positive periodic solution; then positive periodic solutions of Model (1) under state-dependent impulsive conditions are called positive periodic solution generated by impulses.

Remark 10. As we know, the previous papers concerning state-dependent impulsive effect all assumed that the predator just lived on the prey; here we point out that the predator has some other food resources; this is more practical. On the other hand, the existing state-dependent impulsive differential systems mainly discussed the properties of solutions, including existence, uniqueness, and orbitally asymptotical stability. Here, not aiming at the properties of solutions, we 


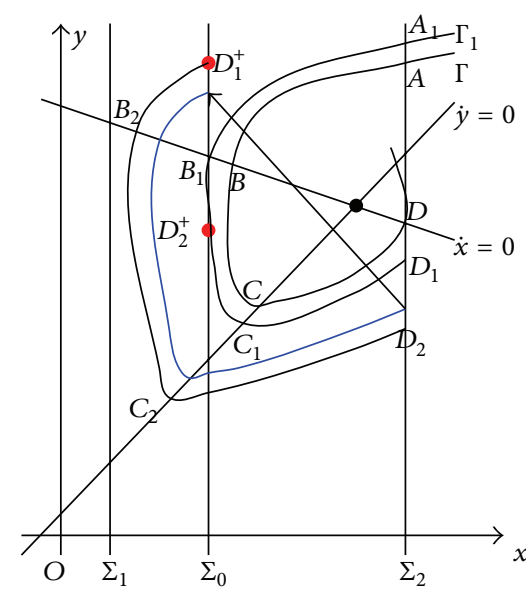

(a)

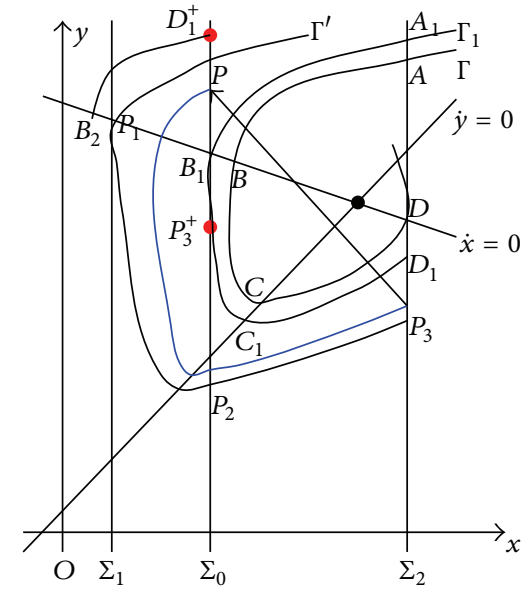

(b)

FIGURE 4: The possible trajectories in the case of $h_{1}<(1-\alpha) h_{2}<x_{B}<x^{*}<h_{2}$.

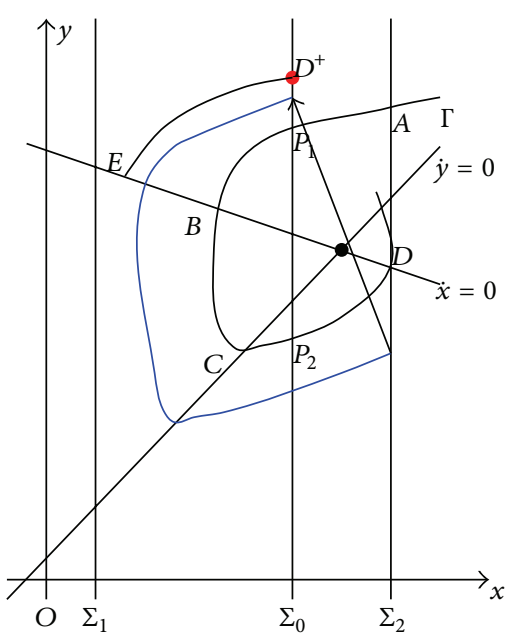

(a)

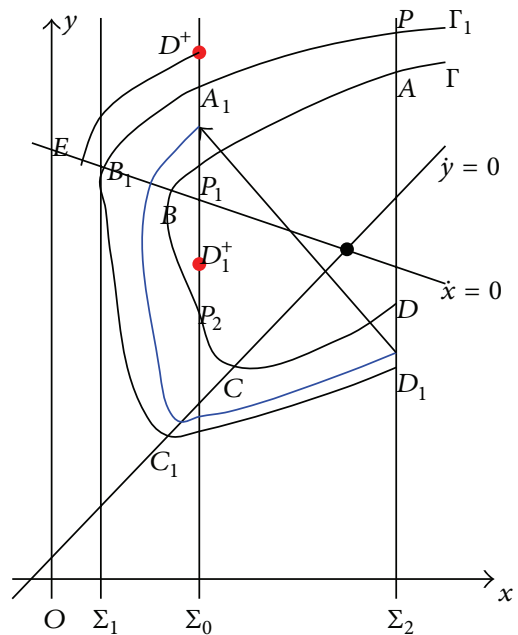

(b)

Figure 5: The possible trajectories in the case of $h_{1}<x_{B}<(1-\alpha) h_{2}<x^{*}<h_{2}$.

are focused on considering the influence of impulsive effect on the system itself. The theoretical results imply that if impulses do not exist, then the predator and prey species will tend to a point; if impulsive effect occurs, then the predator and prey species will be maintained at a periodic oscillation; that is, both the densities of these two species can change periodically. Therefore, our results demonstrate that impulsive effect takes an important role in ecological system.

\section{Simulations and Conclusions}

In this paper, we propose and analyse a state-dependent impulsive predator-prey model in which the predator species display a logistic growth. By using geometrical analysis methods, the existence of positive periodic solutions of Model (1) is given. Here we should point out that the positive periodic solutions are generated by impulses. For system (2), which does not exist as impulsive effect, the interior equilibrium is globally asymptotically stable, the phase trajectory and time series chart can be seen in Figures 6 and 7; therefore, system (2) does not exist as positive periodic solution and all the phase trajectories will tend to the interior equilibrium. When the impulsive effects are operated, system (1) can be gotten, the theoretical results demonstrate that system (1) exists as positive periodic solutions for some cases, and the numerical simulations also illustrate the existence of the periodic solutions; please see Figures 8, 9, and 10. Here $r_{1}=$ $0.4, r_{2}=0.1, a_{11}=0.6, a_{22}=0.6, a_{12}=0.6$, and $a_{21}=0.4$. Therefore, the positive periodic solutions are generated by impulses. 


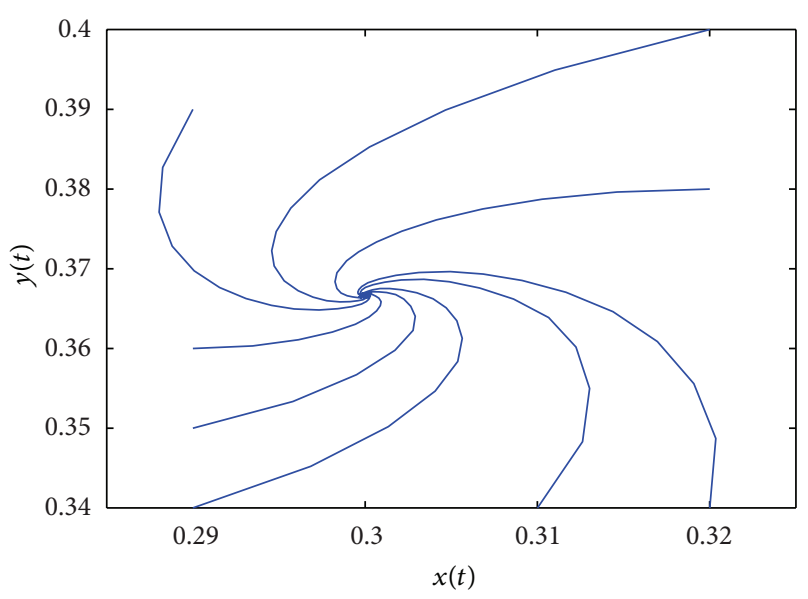

FIGURE 6: The phase trajectory without impulse.

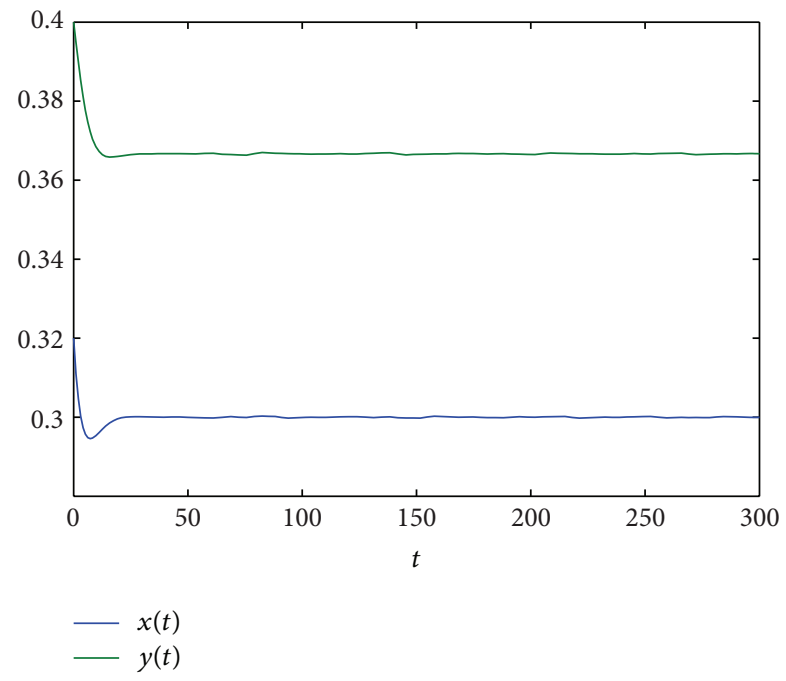

FIGURE 7: The time series chart for $x$ and $y$ without impulse.

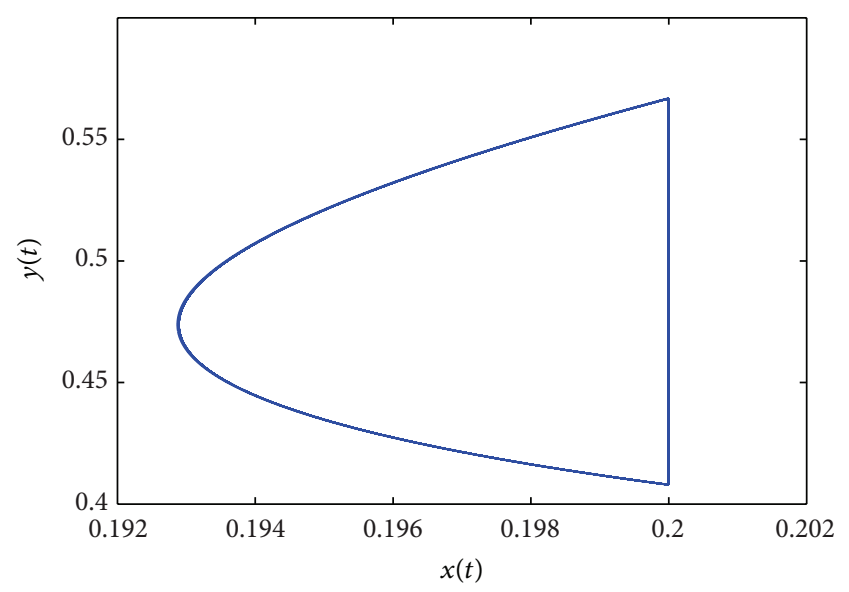

FIgURE 8: Plots of the positive periodic solution when $(1-\alpha) h_{2}<$ $h_{1}<h_{2}<x^{*}$ with $h_{1}=0.2, h_{2}=0.28, \alpha=0.4, \beta=0.2$, and $\tau_{1}=0.16$.

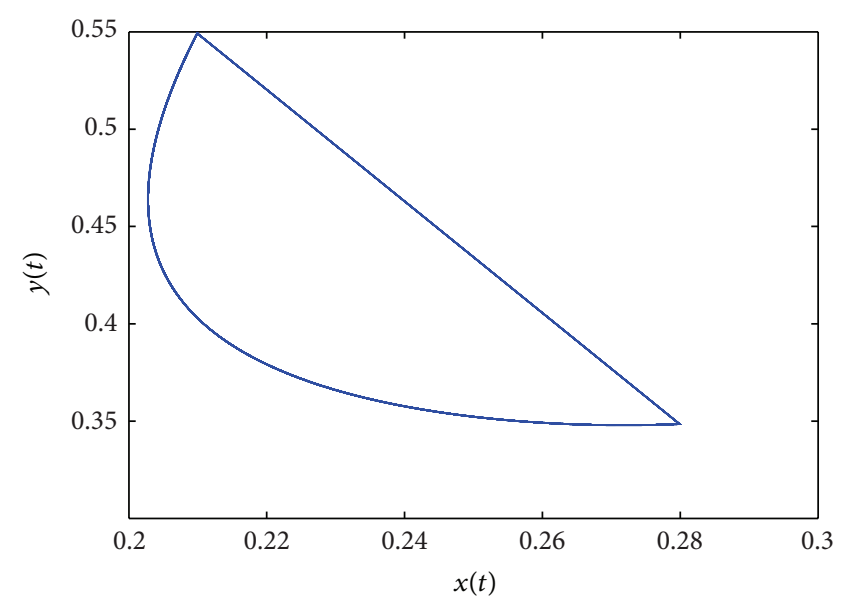

FIgURE 9: Plot of the positive periodic solution when $h_{1}<(1-\alpha) h_{2}<$ $h_{2}<x^{*}$ with $h_{1}=0.2, h_{2}=0.28, \alpha=0.25, \beta=0.2$, and $\tau_{2}=0.27$.

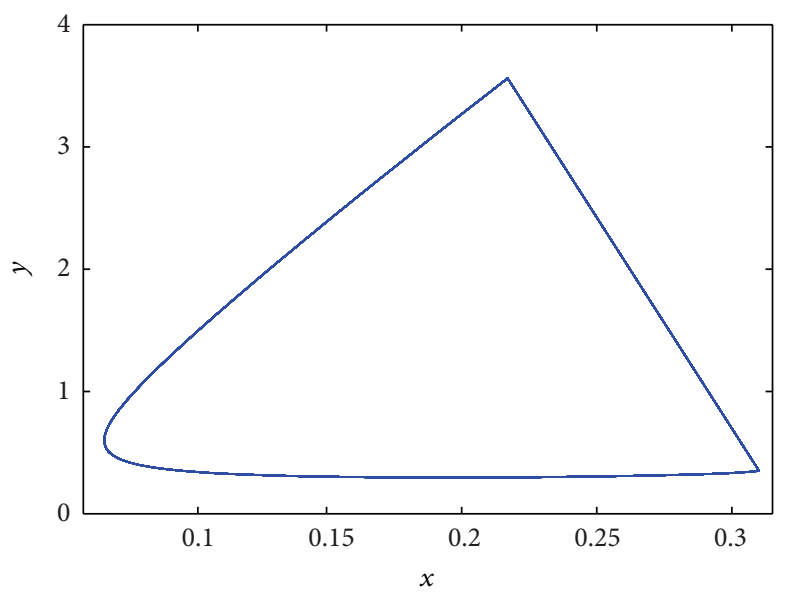

FIgUre 10: Plot of the positive periodic solution when $h_{1}<(1-$ $\alpha) h_{2}<x^{*}<h_{2}$ with $h_{1}=0.05, h_{2}=0.31, \alpha=0.3, \beta=0.2$, and $\tau_{2}=3.28$.

\section{Conflict of Interests}

The authors declare that there is no conflict of interests regarding the publication of this paper.

\section{Acknowledgments}

Qizhen Xiao was partially supported by the Fundamental Research Funds for the Central Universities of Central South University (no. 2013zzts006). Binxiang Dai was partially supported by the National Natural Science Foundation of China (no. 11271371 and no. 51479215).

\section{References}

[1] Y. Z. Pei, C. G. Li, L. S. Chen, and C. Wang, "Complex dynamics of one-prey multi-predator system with defensive ability of prey and impulsive biological control on predators," Advances in Complex Systems, vol. 8, no. 4, pp. 483-495, 2005. 
[2] C.-Y. Huang, Y.-J. Li, and H.-F. Huo, "The dynamics of a stage-structured predator-prey system with impulsive effect and Holling mass defence," Applied Mathematical Modelling, vol. 36, no. 1, pp. 87-96, 2012.

[3] G. Zong, S. Xu, and Y. Wu, "Robust $H_{\infty}$ stabilization for uncertain switched impulsive control systems with state delay: an LMI approach," Nonlinear Analysis. Hybrid Systems, vol. 2, no. 4, pp. 1287-1300, 2008.

[4] J. Guo, C. Liu, and Z. Xiang, "Robust finite-time $H_{\infty}$ control for impulsive switched nonlinear systems with state delay," Mathematical Problems in Engineering, vol. 2012, Article ID 830154, 25 pages, 2012.

[5] X. Li, Z. R. Xiang, and H. R. Karimi, "Asynchronously switched control of discrete impulsive switched systems with time delays," Information Sciences, vol. 249, pp. 132-142, 2013.

[6] S. Huang, Z. Xiang, and H. R. Karimi, "Input-output finite-time stability of discrete-time impulsive switched linear systems with state delays," Circuits, Systems, and Signal Processing, vol. 33, no. 1, pp. 141-158, 2014.

[7] J. H. Chen, C. C. Tisdell, and R. Yuan, "On the solvability of periodic boundary value problems with impulse," Journal of Mathematical Analysis and Applications, vol. 331, no. 2, pp. 902912, 2007.

[8] W. B. Wang, J. H. Shen, and J. J. Nieto, "Periodic boundary value problems for second order functional differential equations," Journal of Applied Mathematics and Computing, vol. 36, no. 12, pp. 173-186, 2011.

[9] J. Chu and J. J. Nieto, "Impulsive periodic solutions of firstorder singular differential equations," Bulletin of the London Mathematical Society, vol. 40, no. 1, pp. 143-150, 2008.

[10] B. X. Dai and D. Zhang, "The existence and multiplicity of solutions for second-order impulsive differential equations on the half-line," Results in Mathematics, vol. 63, no. 1-2, pp. 135149, 2013.

[11] H. Zhang and Z. X. Li, "Periodic and homoclinic solutions generated by impulses," Nonlinear Analysis: Real World Applications, vol. 12, no. 1, pp. 39-51, 2011.

[12] B. Dai, Y. Li, and Z. Luo, "Multiple periodic solutions for impulsive Gause-type ratio-dependent predator-prey systems with non-monotonic numerical responses," Applied Mathematics and Computation, vol. 217, no. 18, pp. 7478-7487, 2011.

[13] D. Chen and B. Dai, "Periodic solutions of some impulsive Hamiltonian systems with convexity potentials," Abstract and Applied Analysis, vol. 2012, Article ID 616427, 8 pages, 2012.

[14] W. C. Zhao, T. Q. Zhang, X. Z. Meng, and Y. Yang, "Dynamical analysis of a pest management model with saturated growth rate and state dependent impulsive effects," Abstract and Applied Analysis, vol. 2013, Article ID 204642, 18 pages, 2013.

[15] H. Cheng, F. Wang, and T. Zhang, "Multi-state dependent impulsive control for pest management," Journal of Applied Mathematics, vol. 2012, Article ID 381503, 25 pages, 2012.

[16] M. Z. Huang, S. Z. Liu, X. Song, and L. Chen, "Periodic solutions and homoclinic bifurcation of a predator-prey system with two types of harvesting," Nonlinear Dynamics, vol. 73, no. 1-2, pp. 815-826, 2013.

[17] C. J. Wei and L. S. Chen, "Homoclinic bifurcation of preypredator model with impulsive state feedback control," Applied Mathematics and Computation, vol. 237, pp. 282-292, 2014.

[18] C. Dai, M. Zhao, and L. Chen, "Homoclinic bifurcation in semicontinuous dynamic systems," International Journal of Biomathematics, vol. 5, no. 6, Article ID 1250059, 19 pages, 2012.
[19] C. Wei and L. Chen, "Heteroclinic bifurcations of a preypredator fishery model with impulsive harvesting," International Journal of Biomathematics, vol. 6, no. 5, Article ID 1350031, 15 pages, 2013.

[20] C. J. Wei and L. S. Chen, "Periodic solution and heteroclinic bifurcation in a predator-prey system with Allee effect and impulsive harvesting," Nonlinear Dynamics, vol. 76, no. 2, pp. 1109-1117, 2014.

[21] L. Zhao, L. Chen, and Q. Zhang, "The geometrical analysis of a predator-prey model with two state impulses," Mathematical Biosciences, vol. 238, no. 2, pp. 55-64, 2012.

[22] C. Dai, M. Zhao, and L. Chen, "Bifurcations and periodic solutions for an algae-fish semicontinuous system," Abstract and Applied Analysis, vol. 2013, Article ID 619721, 11 pages, 2013.

[23] C. Wei and L. Chen, "Periodic solution of prey-predator model with Beddington-DeAngelis functional response and impulsive state feedback control," Journal of Applied Mathematics, vol. 2012, Article ID 607105, 17 pages, 2012.

[24] L. S. Chen, Nonlinear Biological Dynamic Systems, Academic Press, New York, NY, USA, 1993.

[25] V. Lakshmikantham, D. D. Bainov, and P. S. Simeonov, Theory of Impulsive Differential Equations, World Scientific Publishing, Singapore, 1989.

[26] D. Bainov and P. Simeonov, Impulsive Differential Equations: Periodic Solutions and Applications, Longman Scientific and Technical, New York, NY, USA, 1993.

[27] L. S. Chen, "Theory and application of semi-continuous dynamical system," Journal of Yulin Normal University (Natural Science), vol. 34, pp. 1-9, 2013 (Chinese). 


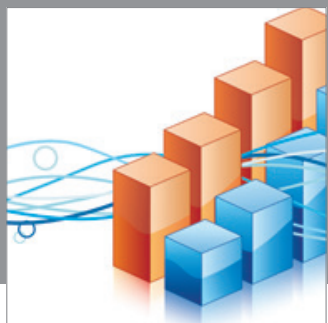

Advances in

Operations Research

mansans

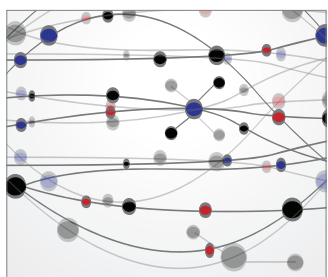

The Scientific World Journal
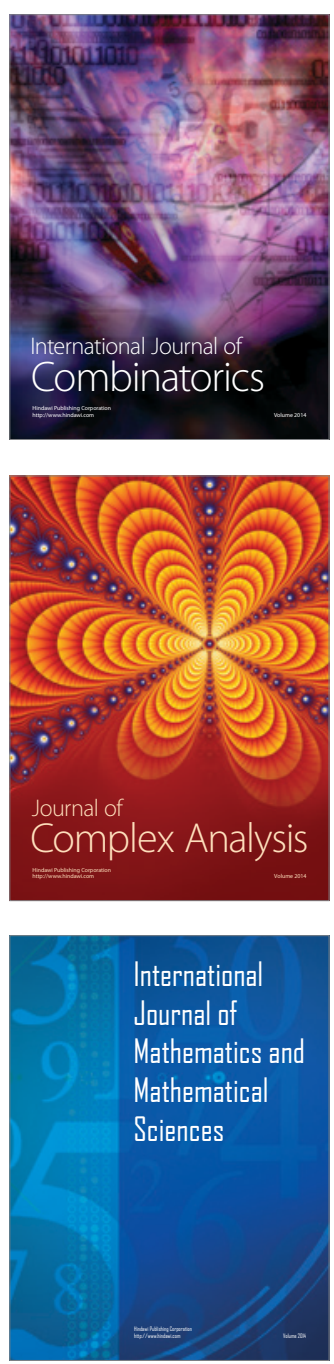
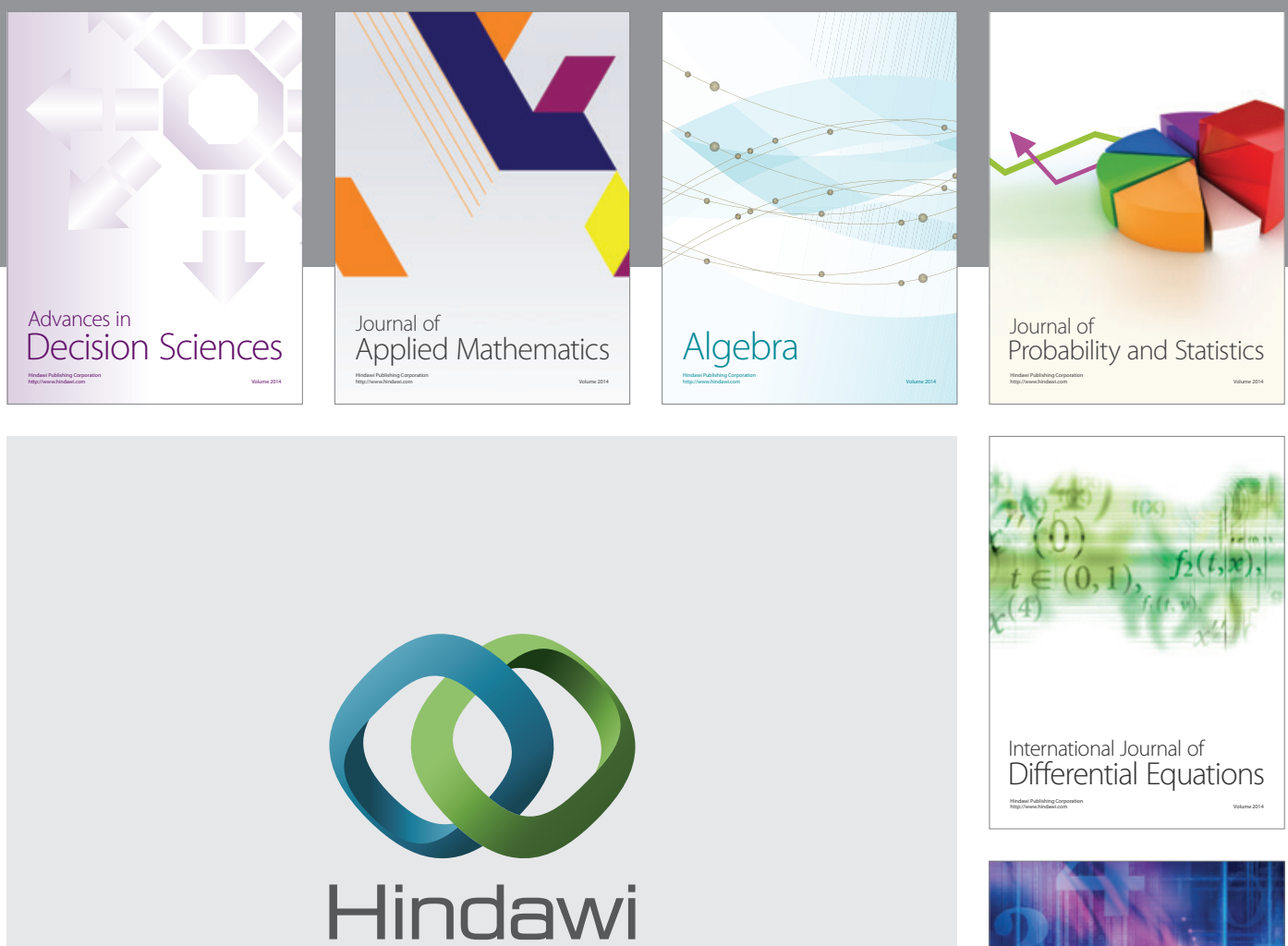

Submit your manuscripts at http://www.hindawi.com
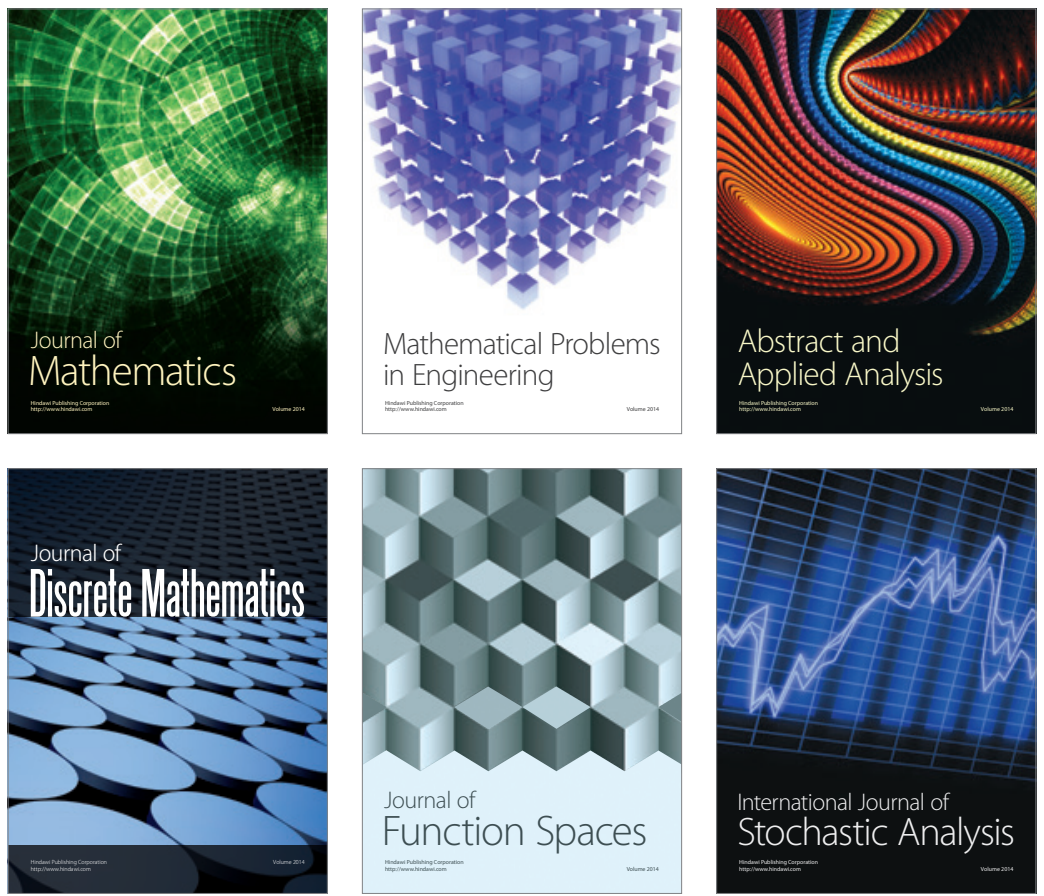

Journal of

Function Spaces

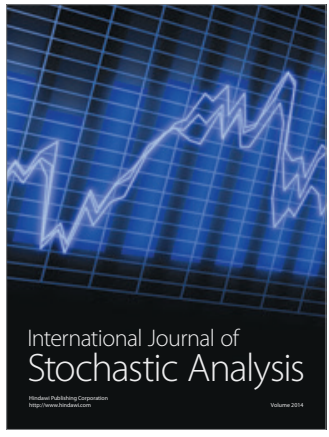

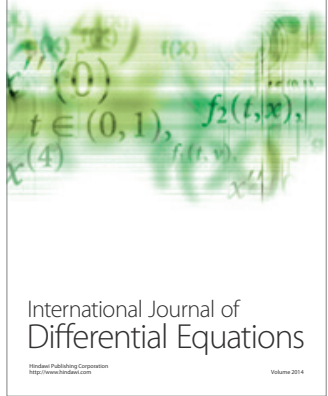
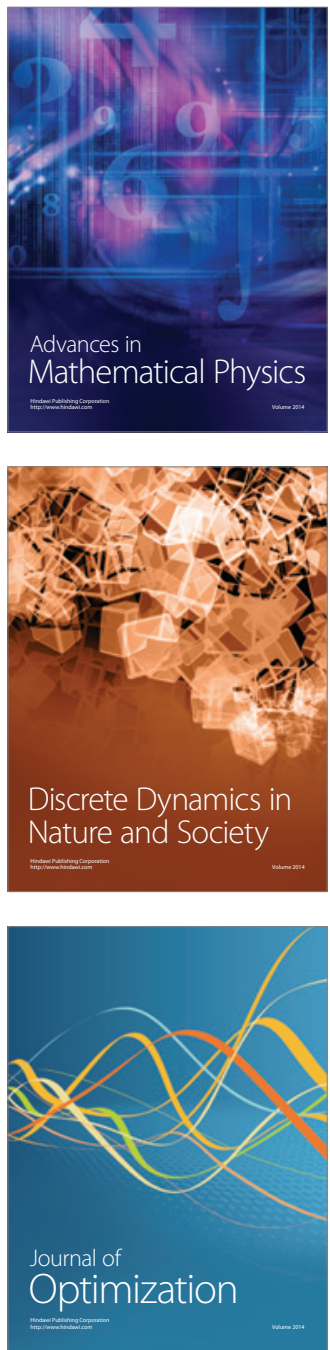